\title{
Metacarpophalangeal Joint 5
}

National Cancer Institute

\section{Source}

National Cancer Institute. Metacarpophalangeal Joint 5. NCI Thesaurus. Code C102320.

A condyloid synovial joint within the fifth digit of the hand connecting the metacarpal to the proximal phalanx. 